\title{
Migración internacional y crisis global: una visión histórico-estructural
}

\author{
Carmen Lilia Cervantes-Bello
}

Universidad del Caribe, México

\begin{abstract}
Resumen
El presente trabajo tiene por objeto brindar un marco de referencia teórico-conceptual del tema migratorio en un contexto histórico y particularmente en una época de recurrentes crisis económicas y financieras. El análisis comienza con un panorama de las migraciones en la era global y la presentación de referentes teóricos que vinculan la globalización con la configuración de movimientos migratorios internacionales. Adicionalmente, se propone analizar la migración desde la perspectiva de reproducción de la sociedad, con la intención de hacer evidentes las contradicciones y tensiones que surgen de los procesos de acumulación, tanto en la forma social como productiva. Se presenta como categoría de análisis el patrón de acumulación y se revisa la evolución de las etapas históricas de acumulación capitalista, así como los efectos de las crisis en los flujos migratorios.
\end{abstract}

Palabras clave: Migración internacional, globalización, crisis, patrón migratorio.

International migration and global crisis: a historical- structuralism approach

\section{Abstract}

This paper presents a conceptual framework for analyzing international migration in a historical context and particularly in a time of recurrent economic and financial crises. The analysis starts with a panorama of migrations in the global era and the presentation of theoretical references which link globalization with the configuration of international migratory movements. Additionally, we propose to analyze migration as a factor in the reproduction and transformation of society. The accumulation pattern is presented as a category of analysis and a review is made of the evolution of the historical stages of capitalist accumulation in order to the migratory phenomenon.

Key words: International migration, globalization, crisis, migration patterns.

Recibido: 14 de enero de 2019

Aprobado: 22 de abril de 2019 


\section{INTRODUCCIÓN}

$\mathbf{L}$

a migración forma parte integral de la economía mundial a pesar de que sus flujos son menores que los producidos en otros momentos históricos. No obstante, las condiciones de un nuevo orden mundial y las tensiones geopolíticas de los últimos tiempos, han derivado en una hiperpolitización negativa del fenómeno migratorio y en el ascenso de fuerzas populistas antiinmigrantes. Sin duda, nos enfrentamos ante una epoca migratoria que tiene nuevas características; en este contexto, la migración se percibe como un fenómeno caótico y los migrantes son señalados como perturbadores del "orden". Los gobiernos tienen la sensación de haber perdido el control de las fronteras, situación que exacerva los nacionalismos y promueve la adopción de políticas de inmigración crecientemente restrictivas en los polos receptores de migrantes.

La idea de un "caos" migratorio se fundamenta hasta cierto punto, en que en estos momentos la proporción de trabajadores indocumentados en los flujos migratorios parece estar por encima de la migración legal. En este sentido, la segmentación entre migrantes cualificados y menos cualificados se ha agudizado, se ha producido un fuerte cuestionamiento y retroceso del derecho de asilo y la preferencia por la migración temporal ha ido en aumento; sin embargo, las tendencias indican que la migración sigue creciendo de manera sostenida sin ser masiva. De acuerdo con Wiliam Lacy (2018) tan solo el 3.5 por ciento de la población mundial son migrantes y producen el nueve por ciento del Producto Interno Bruto (PIB) a nivel global, lo que representa un cuatro por ciento más que lo que producirían si se quedaran en sus lugares de origen; por lo tanto, no estamos ante un fenómeno explosivo y fuera de control.

De acuerdo con Wallerstein (2002: 7), en la base de los flujos migratorios se encuentra una distribución específica de los países, cuyas economías están integradas en un sistema que divide la economía mundial en áreas que se benefician de la acumulación de capital (centrales) y aquellas en constante desventaja por el proceso desigual (periféricas). En este orden de ideas, la migración es un elemento estructural del desarrollo de las sociedades capitalistas, que se activa como 
resultado de las contradicciones y graves desigualdades que genera el sistema, y que cambia de características de acuerdo con los requerimientos de los patrones de acumulación, adquiriendo funciones determinadas y características enmarcadas en la permanencia y funcionamiento del sistema (Aragonés y Dunn, 2005: 46).

No obstante, las migraciones tienen un efecto más amplio como componente y artífice de la reproducción y transformación de la sociedad global, que va más alla de la perspectiva económica. Por lo tanto, es necesario incorporar otras dimensiones que contribuyen a la reproducción como pueden ser: los aspectos demográficos, políticos, sociales, históricos, entre otros, que también reproducen estructuras de clase, estructuras sociales de diferenciación y desigualdad (Canales, 2015: 79). Desde esta perspectiva, es posible entender como la migración internacional activa mecanismos que articulan e integran en un mismo proceso, las condiciones y dinámicas de la reproducción social de los países de origen con las condiciones y dinámicas de la reproducción social de los países de destino.

Por otro lado, es preciso señalar que el desarrollo del capitalismo no ha sido un proceso lineal, ya que siempre ha estado sometido a fluctuaciones cuya principal expresión son las crisis. Si se hace un recorrido histórico de lo sucedido en las crisis del siglo pasado, es factible observar que fueron precisamente crisis estructurales las que iniciaron nuevos patrones de acumulación y que a su vez dieron pie al surgimiento de diversos patrones migratorios. Otro hecho observable, es que los obstáculos a los flujos migratorios son característicos de momentos históricos en los que se intentó salir de la crisis, poniendo en marcha políticas proteccionistas y nacionalistas, tal como sucedió en la primera posguerra, y después de la Gran Depresión. Sin embargo, si las deportaciones y los retornos forzosos han constituido una herramienta histórica de gestión de los flujos migratorios, ¿Qué elementos hacen distinta a la reciente crisis global de 2008?

Como ya se ha señalado, por un lado destaca el neoconservadurismo a partir de un discurso altamente proteccionista, antiinmigrante y de militarización de las fronteras, que se materializa hoy en día con el ascenso de Donald Trump al poder y fenómenos como el Brexit que debilitan la integración al interior de la Unión Europea; debido en parte a la mal llamada crisis de los refugiados. Por otro lado, destaca el lugar que ha tomado la migración calificada como respuesta a las nuevas exigencias de los mercados de trabajo de los países inmersos en 
diversos procesos de desarrollo, como aquellos denominados economía del conocimiento, aspecto que ha provocado una reconfiguración de los flujos migratorios a nivel mundial y una competencia global por los talentos.

Derivado de lo anterior, el presente trabajo tiene por objeto brindar un marco de referencia conceptual del tema migratorio en un contexto histórico y particularmente en una época de recurrentes crisis económicas y financieras. Para tal efecto, el análisis comienza con un panorama de las migraciones en la era global y la presentación de referentes teóricos que vinculan la globalización con la configuración de movimientos migratorios internacionales, poniendo énfasis en los trabajos de Saskia Sassen y David Harvey, los cuales permiten reforzar la idea de que los desplazamientos humanos que se producen a partir del desarrollo capitalista, presentan un comportamiento específico asociado a las transformaciones de las diferentes fases de acumulación. De igual manera, se propone analizar la migración desde la perspectiva de reproducción de la sociedad, con la intención de hacer evidentes las contradicciones y tensiones que surgen de los procesos de acumulación, tanto en la forma social como productiva. Situación que permite resaltar un componente central del enfoque estructural y del marxismo en particular: la contradicción como motor de acumulación.

Posteriormente, se presenta como categoría de análisis el patrón de acumulación y se realiza un repaso de la evolución de las etapas históricas de acumulación capitalista en función del fenómeno migratorio y se incorpora un elemento adicional: las crisis; ya que se consideran bisagras entre una época y otra y el punto de inflexión que marcan el surgimiento de nuevas etapas de acumulación. Por consiguiente, la migración de trabajadores se expande o contrae en función de los requerimientos del capital. Si bien, a lo largo de la historia se han presenciado múltiples crisis, solamente se analizan las más trascendentales (la Gran Depresión, la gran recesión de los años 70's y la crisis estructural de 2007-2008) y sus efectos en los flujos migratorios. Finalmente se presentan las conclusiones.

\section{LAS MIGRACIONES EN LA ERA GLOBAL}

De acuerdo con Saskia Sassen (2003: 87) "las migraciones internacionales son parte de procesos sociales, económicos y políticos más amplios. Si bien los individuos experimentan la migración como resultado de sus decisiones personales, la opción de migrar es producida 
socialmente". Tal afirmación permite asumir que la migración es un fenómeno global, que guarda una alta complejidad estructural y que requiere de explicaciones multifactoriales. A través del tiempo las diversas causas de los desplazamientos, así como sus características y consecuencias, han generado distintos tipos de migración. A pesar de que las tendencias indican que la migración continua creciendo de manera sostenida sin ser masiva, existen tres tipos de migraciones que se han intensificado en las últimas décadas.

En primer lugar, destaca la migración indocumentada como resultado de las disparidades socioeconómicas, la creciente restricción a la movilidad legal y la complementariedad demográfica norte-sur. ${ }^{1}$ En segundo lugar, se encuentran las migraciones por violencia, conflictos (sociales y políticos) y catástrofes naturales; ${ }^{2}$ y finalmente, en tercer lugar, el aumento de las migraciones internas que involucran a más de 750 millones de personas y que se dan como resultado de la creciente urbanización ${ }^{3}$ del planeta, aspecto que repercute en los mercados laborales, el crecimiento de la informalidad y por supuesto, en la emigración internacional (Lacy, 2018).

Sin embargo, un rasgo distintivo de las migraciones en la actualidad es su "carácter global y dinámico en términos de la dirección de los flujos, su composición y modalidades" (Artola, 2016: 25). La migración es global porque involucra prácticamente a todos los países del mundo de una u otra forma, ya sea como países de origen, de destino o de tránsito, y a veces incluso presentando dos o tres de estas características simultáneamente; pero también porque la migración es un componente clave de los cambios globales del mercado laboral, aspecto que repercute a nivel político, económico y social en casi todos los países, aunque con distintos niveles de intensidad. Por lo tanto, el

1 Esta complementariedad implica que, generalmente en los países del Norte hay una escasez relativa de fuerza de trabajo, debido a que la población económicamente activa no puede mantenerse con bajas tasas de crecimiento poblacional. Adicionalmente existen otras necesidades que requieren fuerza de trabajo adicional, que pueden ser de carácter coyuntural (expansión de la economía), temporal o sectorial (actividades mal remuneradas o peligrosas; servicios en expansión como el cuidado de niños o adultos mayores, jardinería, servicio doméstico, etc.) Si bien, en este punto del análisis se emplea el término complementariedad, es un hecho que la migración internacional contribuye a la configuración de un modelo de reproducción demográfica propio de la globalización, que no está exento de tensiones y contradicciones. Aspectos que se desarrollan más adelante.

2 Estimaciones del Banco Mundial indican que para el 2050, los impactos del cambio climático podrían provocar el desplazamiento de más de 140 millones de personas dentro de sus respectivos países. (World Bank, 2018) Para consultar el documento completo ingresar a: https://openknowledge.worldbank.org/handle/10986/29461

3 Para William Lacy, Director General de la OIM, la urbanización junto a la migración y la diversidad es una de las tres megatendencias del siglo XXI. 
fenómeno migratorio se ha convertido en uno de los principales temas de la agenda global en un intento de gestionar los flujos migratorios de forma integral y a escala internacional.

Asimismo, los migrantes ya no se desplazan predominantemente del sur al norte, puesto que la direccionalidad de los flujos se ha transformado en tres sentidos: sur-sur, norte-norte y norte-sur. De acuerdo con Artola (2016: 25) un tercio se desplaza efectivamente del sur al norte, menos de un tercio se desplaza entre los países del sur y más de un tercio se desplaza o bien entre los países del norte (aproximadamente el 25 por ciento) o desde el norte al sur (menos del 10 por ciento). Vale la pena señalar, que si bien es cierto que los trabajadores migrantes se han visto forzados a desplazarse para superar las carencias económicas y las escasas oportunidades de mejora en sus propios países, "existe una considerable fuerza estructural de atracción que se da en los mercados laborales de los países desarrollados" (Artola, 2016: 26), ya que al mismo tiempo satisfacen las demandas y exigencias de los mercados de trabajo de los países que se encuentran inmersos en diversos procesos de desarrollo, como aquellos denominados economía del conocimiento. ${ }^{4}$

Las modalidades de la migración también han cambiado, además de la migración tradicional con fines laborales, es preciso distinguir algunas modalidades nuevas como la migración por motivos familiares, la creciente feminización de la migración, así como el aumento de la migración infantil y de la migración forzada, ${ }^{5}$ la cual caracteriza a una parte sustancial de la dinámica migratoria contemporánea. Adicionalmente, como respuesta a la crisis global de 2008 se dan las migraciones de retorno, lo que implica nuevos retos económicos, sociales e incluso ambientales que deben enfrentar las localidades de acogida.

Un rasgo común en la proliferación de estas nuevas modalidades migratorias en el plano internacional, es que "son producto de profundas transformaciones productivas, tecnológicas, institucionales y, más

4 De acuerdo con el Banco Mundial este tipo de economía se centra en el conocimiento, ya que se considera que es un activo más importante que los bienes de capital y mano de obra, por ende, la cantidad y sofisticación del conocimiento que permea en las actividades económicas y sociales, llega a niveles muy altos (World Bank, 2016). Para más información consultar http://go.worldbank.org/AW9KZWJB10

5 En los últimos años se le ha otorgado una connotación más amplia a este término. Para Delgado, Márquez y Puentes (2013) se trata de personas literalmente expulsadas de sus territorios, que se desplazan a otros lugares por necesidad y no de manera libre y voluntaria, con la esperanza de acceder a medios de subsistencia u oportunidades de movilidad social, ya sea en su país o fuera de él; o bien de personas que no encuentran condiciones de empleo acordes a su capacidad y a su formación en sus lugares de origen. 
visiblemente, en la división del trabajo; estas ocurren más allá de marcos políticos, normativos e institucionales que buscan administrarlas" (Martinez, 2000: 23). Aquí es preciso señalar que, durante la última década del siglo pasado algunos países altamente industrializados como Estados Unidos, comenzaron a experimentar importantes transformaciones en sus procesos productivos. Ante un nuevo régimen de acumulación y paradigma tecnológico, los mercados de trabajo internacionales modificaron sus características para satisfacer los requerimientos de la producción, lo que a su vez derivó en nuevas condiciones para la mano de obra migrante vinculada con dichos mercados.

De acuerdo con Aragonés y Salgado (2011: 80), si bien las características laborales presentan diferencias, su funcionalidad es la misma y está relacionada con las diferencias en el costo laboral unitario de los trabajadores extranjeros, lo que permite a los países receptores ser más competitivos. Esta reorganización del trabajo, por un lado demanda una mano de obra cada vez más calificada con características específicas que le permita incorporarse a esas nuevas formas de producción; y por el otro, contempla una importante participación de mujeres y trabajadores indocumentados, además de nuevos marcos normativos para todos los trabajadores migrantes.

Cabe señalar que, en el pasado la óptica para abordar las cuestiones asociadas a la migración se circunscribía a un espacio nacional, unilateral y en última instancia, al plano bilateral. La intensificación de las relaciones sociales y la idea de una dimensión y de un mundo global, no constituían hasta hace pocos años un tema habitual en la agenda de los gobiernos y sociedades civiles. Para Artola (2016: 36), a partir de la década de los 90's se inició un proceso de multilateralización del tema migratorio, al establecerse mecanismos regionales de diálogo y cooperación de carácter no vinculante, con el fin de dar respuesta a aspectos complejos y delicados. En el proceso, comenzaron a involucrarse nuevos actores relevantes como las organizaciones de la sociedad civil, redes transfronterizas, sindicatos, organismos internacionales, entre otros.

Derivado de lo anterior y en concordancia con Martínez Pizarro (2000: 29) es preciso considerar que:

La globalización "es un conjunto de procesos complejos que la sitúan como un fenómeno de influencia decisiva sobre la percepción y evolución de la migración internacional, si se acepta que ésta constituye un proceso social de múltiples dimensiones. 
Sin embargo, ¿Qué es lo que se entiende por globalización? Si bien las formaciones globales han existido desde hace siglos, en la actualidad tienen un carácter muy diverso que plantea ciertos desafíos teóricos, debido a que lo global reside al interior de los territorios e instituciones nacionales.

Para Saskia Sassen (2007: 7) la globalización se trata de dos dinámicas diferenciadas: por un lado, la formación de procesos e instituciones explícitamente globales, como pueden ser las organizaciones y tribunales internacionales, los mercados financieros internacionales, las redes empresariales globales o el nuevo cosmopolitismo. ${ }^{6}$ Por otro lado, están aquellos procesos que no pertenecen a la escala global y que, sin embargo, forman parte de la globalización. Esto significa que, aunque se localicen en ámbitos nacionales o subnacionales, forman parte de ésta, porque incorporan redes o entidades fronterizas, que conectan múltiples procesos y actores con la esfera global; o bien porque se trata de dinámicas que se registran en un número cada vez mayor de países.

Para ejemplificar mejor la segunda dinámica, es posible hacer referencia a las redes transfronterizas ${ }^{7}$ dedicadas a alguna causa local que también se da a escala global, como lo es el caso de las organizaciones de defensa de los derechos humanos de los migrantes. ${ }^{8}$ Otro ejemplo, es el hecho de que los países estén comenzado a diseñar y utilizar instrumentos internacionales para resolver aspectos que antes habrían resuelto exclusivamente con instrumentos nacionales. En cuestiones migratorias, es preciso mencionar el Pacto Mundial para la Migración el cual busca ser un marco unificador de principios, compromisos y entendimientos comunes entre los países miembros sobre aspectos de la migración internacional. Al respecto Sassen (2007: 6) señala que, generalmente las ciencias sociales estudian la globalización desde los fenómenos explícitamente globales y no se concentran en el segundo tipo de dinámicas que se acaban de señalar. Por lo tanto, para una mejor comprensión de la complejidad y diversidad de los procesos globales las nociones de territorio, escala y los distintos significados de

6 En la actualidad, existe una gran cantidad de culturas originales de un pueblo a país en particular que se han asentado en ciudades como Londres, Tokio o Nueva York dando paso a nuevos tipos de cosmopolitismo.

7 Aquí se hace referencia a una variedad de actores y actividades cada vez más amplia como son la red de filiales de una empresa, las redes de terrorismo internacional o las redes transnacionales de inmigrantes.

8 Los dreamers crearon la Red Solidaria Transfronteriza de Jóvenes Activistas Migrantes que representa a miles de latinoamericanos para exigir sus derechos en sus países de origen, de tránsito, destino y retorno. 
lo nacional son categorías esenciales, sobre todo porque se relacionan con tres objetos de estudio tradicionales para la sociología: las estructuras, las prácticas y las instituciones (Sassen, 2007: 25, 26).

Otra característica importante de los procesos globales en la actualidad es su carácter multiescalar. ${ }^{9}$ En este sentido, el proyecto global de las grandes empresas, las nuevas capacidades que resultan de las tecnologías de la información y el crecimiento de componentes supranacionales en la labor del Estado, son ejemplos de la construcción de nuevas escalas que van más allá de lo nacional. Esto no significa que las viejas jerarquías hayan desaparecido, sino que, junto a las anteriores surgen nuevas prácticas y procesos que desestabilizan parcialmente la jerarquía preexistente como expresión de las relaciones de poder y la economía política (Sassen, 2007: 44). Por ejemplo, el espacio operativo de una empresa multinacional conlleva una configuración multiescalar compleja, ya que entre sus elementos fundamentales se encuentran tanto la red de filiales en distintas regiones del planeta, como la concentración de funciones estratégicas en una sola ubicación. De igual manera, mientras más se globalizan y digitalizan las operaciones y los mercados globales, más complejas y estratégicas se vuelven las funciones de gestión y servicios especializados, característica principal de las ciudades globales. ${ }^{10}$

Desde esta perspectiva, la globalización puede deconstruirse en términos de los lugares estratégicos donde se materializan los procesos globales y los vínculos que los conectan. Sin duda, la migración es uno de los procesos constitutivos de la globalización y de una nueva economía política transnacional, tanto en el nivel macro de los mercados laborales globales como en el nivel micro de las estrategias de supervivencia de los migrantes y sus hogares. En este orden de ideas, a continuación se revisan ciertos vínculos teóricos entre globalización y migración internacional, en función de dos autores principales Saskia Sassen $(2003 ; 2007 ; 2008)$ y David Harvey $(2003 ; 2006$; 2013).

9 Hoy en día, al interior de los procesos globales existen ciertas condiciones que no es posible organizar en jerarquías centradas en el estado-nación, se trata más bien de un sistema que opera a través de todas las escalas. No se trata de un simple ascenso de lo local a lo regional y de allí a lo internacional o viceversa.

10 El concepto de ciudad global fue acuñado por Saskia Sassen en 1991 y pone énfasis en algunos de los impactos de la globalización en las ciudades, particularmente la configuración de polos urbanos interconectados globalmente que actúan como potentes ejes de atracción de sector privado y de profesionales del ámbito financiero, tecnológico y de la innovación. 


\section{VÍNCULOS TEÓRICOS ENTRE LA GLOBALIZACIÓN Y LA CONFIGURACIÓN DE MOVIMIENTOS MIGRATORIOS INTERNACIONALES}

Para Saskia Sassen $(2003 ; 2007 ; 2008)$ gran parte de los análisis que intentan explicar la configuración de los movimientos migratorios internacionales se basan en los factores de atracción/expulsión y si bien, son útiles para explicar los casos individuales y las condiciones del sistema en una zona o país determinado, por sí solos, no dan cuenta de una realidad mucho más compleja. Entre los principales factores de expulsión se encuentran la pobreza y el desempleo, mientras que los factores de atracción más importantes son la posibilidad de obtener un empleo y una mejor calidad de vida. En un sentido estricto, dichos factores deberían provocar movimientos migratorios masivos hacía otros países, pero no es así. Visto de esta manera, los factores de atracción/expulsión permiten explicar por qué los individuos emigran, pero no por qué la mayoría de personas que viven en condiciones similares permanecen en sus lugares de origen. Aquí la pregunta clave sería, bajo qué condiciones la pobreza se convierte en un factor de expulsión.

Para la autora, existen variables adicionales que transforman esas condiciones en motivos de emigración, como pueden ser la contratación directa por parte de los empleadores en el país de destino o la decisión familiar de enviar a un miembro del hogar a vivir a otro país. Por lo tanto, centrarse en los casos individuales no es suficiente, si bien es posible considerar que la migración es resultado de decisiones personales, la opción de migrar es un producto social. Este dato, posiblemente se pierde con frecuencia en los análisis porque los movimientos migratorios comparten muchas características:

La mayoría de los inmigrantes provienen de países menos desarrollados que el país receptor y tienen un nivel de ingresos y de educación bajo o medio, lo que a su vez genera la idea de que la pobreza y el desempleo son los principales factores de expulsión. Sin embargo, muchos países con tasas elevadas de pobreza y de desocupación carecen de una tradición migratoria significativa, y en otros la emigración es un fenómeno reciente, aunque la pobreza exista desde hace años (Sassen, 2007: 168).

En este orden de ideas, son varios los aspectos que pueden transformar una situación generalizada de pobreza y desempleo en un factor de expulsión: la interdependencia existente entre una cantidad 
cada vez mayor de países, la formación de espacios transnacionales de actividad económica, las actuales redes globales de tráfico de personas, los lazos entre las antiguas colonias y los países colonizadores, entre otros. Así, en un ejercicio de abstracción de ciertas particularidades, la autora identifica tres tendencias que articulan los movimientos migratorios internacionales con las condiciones fundamentales de la globalización actual:

a) La geoeconomía de las migraciones explica que, gran parte de los movimientos migratorios comienzan mediante una contratación directa de mano de obra por parte de empresas, gobiernos, contratistas o traficantes. Este fenómeno se ha dado en distintos grados durante períodos históricos diferentes y en diversas partes del mundo. Sin embargo, quién contrata y desde dónde lo hace, es un asunto que:

Ha sido moldeado por los múltiples imaginarios globales de la actualidad y por otros vínculos político-económicos previos, como el colonialismo, la inversión extranjera y otras operaciones empresariales en el contexto de la globalización económica (Sassen, 2007: 172).

Por ejemplo, la conformación de un sistema económico transatlántico que vinculó a varios estados-nación mediante alianzas bélicas y transacciones económicas, constituiría la base para el desarrollo estadounidense. Es a partir de la circulación de capital, bienes y mano de obra, que las nuevas migraciones de Europa a América se vieron favorecidas durante el siglo XIX (Rodríguez, 1985). Un análisis similar se podría contemplar para el Tratado de Libre Comercio de América del Norte (TLCAN), ${ }^{11}$ en lo que respecta al aumento de la corriente migratoria de México hacia los Estados Unidos a partir de los años 90's. Así, los patrones de inmigración previamente señalados indican que existe una geoeconomía de las migraciones. Es decir, los países receptores de mano de obra inmigrante fueron ampliando su zona de contratación e influencia a medida que se volvían más ricos y desarrollados. En la actualidad, dicha zona abarca un conjunto cada vez mayor de países y una gran variedad de dinámicas migratorias, ya sea que se basen en las condiciones imperiales del pasado o en asimetrías actuales, que subyacen a gran parte de los flujos migratorios (Sassen, 2007: 174).

11 En el marco de las negociaciones para modernizar el TLCAN, el pasado 30 de noviembre se alcanzó un nuevo acuerdo denominado Tratado entre México, Estados Unidos y Canadá (T-MEC). 
b) Los puentes de contacto se conforman por los lazos generados por la globalización económica y aquellos que surgen de la contratación de trabajadores extranjeros. La primera categoría abarca la fuerte presencia de empresas multinacionales, la internacionalización de la producción, la inversión extranjera y la fuga de cerebros. Un ejemplo de esto sería la implantación de la agricultura para exportación, mediante inversiones en los países en desarrollo, las cuales desplazan las actividades económicas tradicionales y eliminan oportunidades de supervivencia para los pequeños productores quienes migran en busca de trabajo, en un principio dentro de su propia región, pero a largo plazo hacia otros países. Dentro de la segunda categoría se encuentran las redes de contratación laboral y las redes étnicas. La contratación de mano de obra migrante puede darse a través de campañas promovidas por los gobiernos, organizadas directamente por los empleadores, mediante el tráfico ilegal de trabajadores o por la influencia de redes de parentesco y vecindad (Sassen, 2007: 181). Un factor determinante que permite el funcionamiento de dichas redes es la demanda de trabajadores inmigrantes, en mayor medida por parte de las economías avanzadas.

En cuanto a los lazos étnicos entre las comunidades de origen y las comunidades de inmigrantes de los países receptores, suelen materializarse en la formación de familias transnacionales o estructuras de parentesco ampliado; ya que una vez que existe un flujo migratorio, se garantiza su reproducción en el tiempo. En este punto, no se coincide del todo con Sassen, ya que los flujos migratorios no son consecuencia de un proceso acumulativo producto de las redes sociales, más bien, lo que da lugar a su permanencia, es la relación entre los factores de expulsión y los factores de atracción de los países receptores que reciben inversiones extranjeras que ponen en movimiento procesos productivos cuyas demandas laborales son incapaces de satisfacer con los contingentes internos (Aragonés y Dunn, 2005: 59). Por lo tanto, son las condiciones del mercado de trabajo las que expanden los flujos migratorios y no necesariamente las redes sociales.

c) La exportación organizada de mano de obra ya sea legal o ilegal consiste en operaciones realizadas para obtener renta o para incrementar los ingresos públicos. La intención de Sassen (2007: 189) es explicar la relación entre el crecimiento de esa exportación organizada y las condiciones económicas de los países en vías de desarrollo, como pueden ser el aumento del desempleo, el incremento de la deuda 
pública o el cierre de pequeñas y medianas empresas orientadas al mercado interno. En este sentido, los diversos tipos de exportación de mano de obra se han fortalecido en un período en que las dinámicas ligadas a la globalización económica tienen una profunda incidencia sobre los países en vías de desarrollo, quienes se han visto forzados a aplicar un conjunto de políticas bajo la presión de organismos internacionales como el Fondo Monetario Internacional (FMI) y el Banco Mundial (BM), como los planes de ajuste estructural, la apertura comercial, la eliminación de subsidios estatales y las inevitables crisis que estos cambios conllevan. Asimismo, dentro de esta categoría se incluye el tráfico de hombres, mujeres y niños. Cabe señalar, que dicha exportación se ve facilitada por la conformación de mercados globales, el fortalecimiento de las redes transnacionales y translocales, y por el desarrollo de métodos de comunicación tecnológicos que escapan sin dificultad a los medios convencionales de vigilancia.

Cada uno de los vínculos presentados se sitúa en la intersección de sistemas formales y de prácticas concretas. En tal punto, diversas dinámicas globales y desnacionalizadoras adquieren relevancia para ayudar a entender los flujos migratorios actuales. Para la autora, entender cómo la fase actual de las migraciones difiere de fases anteriores en el mundo capitalista implica considerar la implementación de cambios complejos en esas migraciones, en su representación ideológica y en el significado subjetivo que adquiere para los propios migrantes (Sassen, 2007: 204).

Por su parte David Harvey (2003: 79) señala que, la globalización "es un proceso de producción de desarrollo temporal y geográfico desigual". Tal afirmación, no supone que el proceso sea constante ni impide decir, que haya entrado en una fase radicalmente nueva o avanzado hacia una condición particular o definitiva. Para el autor, algo similar a la globalización está presente desde hace tiempo en la historia del capitalismo. Sin embargo, uno de los aspectos que señala "el ascenso a la preeminencia del término globalización es una profunda reorganización geográfica del capitalismo" (Harvey, 2003: 76). Es decir, el capitalismo ha recurrido a la reorganización geográfica como una solución a sus crisis y puntos muertos. Por lo tanto, construye una geografía acorde a sus necesidades; un espacio producido de infraestructura, organizaciones territoriales, transporte y comunicaciones que facilita la acumulación durante una fase de su historia. Dicho espacio tendrá 
que ser reconfigurado para dar paso a más acumulación en una fase posterior.

Al respecto, Harvey (2000: 248) concuerda con Marx (2000) en una de las características sistémicas de la economía capitalista, que es el carácter estructural de la hiperacumulación y las crisis (contradicciones internas). Esto significa que, la sobreacumulación en un territorio determinado se manifiesta cuando existe un excedente simultáneo de capital y mano de obra sin capacidad para realizar ninguna tarea socialmente productiva, aunado a la inserción desigual de los territorios y formaciones sociales en el mercado mundial (Harvey, 2003; Harvey, 2006). Por consiguiente, si se desea evitar un desequilibrio en el capital que afecte a todo el sistema, se debe encontrar la forma de absorber los excedentes: la expansión geográfica y la reorganización espacial son dos opciones posibles. ${ }^{12}$ En este punto, nuevamente coincide con Marx (2000: 249) en la tendencia del capital hacia la expansión extensiva e intensiva y en su impulso de eliminar todas las barreras espaciales annihilation of space throuhg time (aniquilar el espacio a través del tiempo), pero que sólo puede hacer mediante la producción de un espacio adaptado.

De esta manera, el capitalismo produce un escenario geográfico de relaciones espaciales, de organización territorial y de sistemas de lugares vinculados en una división global del trabajo y de las funciones, adecuado a su propia dinámica de acumulación en un momento particular de su historia; escenario que destruye, reconstruye y adapta a la acumulación en una fecha posterior. Esto significa que la acumulación de capital se relaciona con el espacio y crea su propia geografía histórica. Sin embargo, las diferencias geográficas son más que simples legados históricos, ya que son perpetuamente reproducidas, sostenidas y reconfiguradas por los procesos políticos, económicos y sociales que tienen lugar en el presente (Harvey, 2003: 98) y que, sin duda impulsan de forma poderosa el proceso de globalización en el mundo.

Derivado de lo anterior, es factible analizar los flujos de fuerza de trabajo en el contexto de globalización en función de la segmentación geográfica de la producción a escala global, y la relación entre el régimen actual de acumulación y la configuración local del espacio. Como

12 La organización de divisiones territoriales del trabajo totalmente nuevas, la apertura de nuevas y más baratas fuentes de recursos, de nuevos espacios dinámicos para la acumulación de capital, y la penetración de estructuras sociales preexistentes por parte de las relaciones sociales capitalistas y acuerdos institucionales, son formas de absorber excedentes de capital y mano de obra. 
ya se ha señalado, el sistema económico global se manifiesta en una serie de localizaciones concretas. ${ }^{13}$ Las características de estos espacios locales o subnacionales confieren al capital una serie de ventajas comparativas que le permiten desarrollar superioridad estratégica en el marco de la competencia internacional (Hjorth, 2011: 314). Las ventajas comparativas más comunes se relacionan con el precio y las características de la fuerza de trabajo, la ubicación geoestratégica, infraestructura y los marcos normativos.

Desde esta perspectiva, la segmentación geográfica de los procesos productivos implica no solamente el uso de los factores de producción que se encuentran ya presentes en el espacio local donde se insertan, sino que buscan asegurar el suministro oportuno de todos los insumos. Por ejemplo y de modo fundamental, la fuerza de trabajo. Ya sea alentando y favoreciendo la migración desde áreas geográficas distantes, o mediante mecanismos activos que tienden a producir flujos migratorios directamente en los lugares de origen. Así, la migración internacional de trabajadores se considera un componente central de los procesos productivos y un factor que favorece la internacionalización, objetivo histórico del capitalismo.

En concordancia, Aragonés (2000: 20) señala que:

La migración de trabajadores es un componente estructural de la acumulación capitalista, formado por un excedente laboral mundial que se ha ido generando como producto y necesidad del capitalismo y que persiste bajo el signo de la explotación.

Debido a múltiples aspectos, entre los que destaca: I) la profundización de las diferencias en el desarrollo entre países, II) la necesidad de los polos desarrollados de incorporar a los trabajadores migrantes con el objetivo de regular sus mercados de trabajo, III) la incapacidad de los países subdesarrollados para generar políticas económicas efectivas que permitan la absorción laboral de su población en condiciones dignas, y IV) la falta de efectividad de las regulaciones internacionales para establecer mejores condiciones para los trabajadores migratorios.

Lo anterior permite considerar un cambio de léxico de globalización a "desarrollo geográfico desigual" que propone Harvey (2003: 87) y que ha sido retomado en el campo de las migraciones por Raúl Delgado y Humberto Márquez. Para estos autores, el desarrollo desigual:

13 Las ciudades globales, las zonas francas, centros bancarios offshore, etc. 
Encapsula esta dinámica dominante y hace referencia al proceso histórico, económico, social y político de polarización entre regiones, países y clases derivado de la dinámica de acumulación capitalista, división internacional del trabajo, entramado geopolítico y conflicto de clases en distintas esferas espaciales y niveles jerárquicos (2012: 9).

Esto implica el desarrollo de formas específicas de transformar los componentes del espacio local, las estructuras sociales y económicas locales (Hjorth, 2011: 316). En consecuencia, la migración es un elemento estructural del desarrollo de las sociedades capitalistas que se da como resultado de las contradicciones y graves desigualdades que genera el sistema, pero también por las tensiones entre los factores de expulsión-atracción, vinculados a las necesidades de acumulación capitalista.

Sin embargo, para Alejandro Canales (2015: 82) el análisis de la migración no solo se trata de categorías económicas como la fuerza de trabajo y la reproducción de capital, sino que también, debe involucrar la articulación y contraposición de sujetos sociales y su posición desigual en una estructura social. Desde esta perspectiva, la migración adquiere un papel y significado en la reproducción y transformación de la sociedad global, y por tanto, contribuye a su dinámica y continuo devenir. Partiendo de esta premisa, analizar y problematizar la migración en función de sus contribuciones y tensiones respecto a la reproducción de lo social, puede abordarse desde tres dimensiones: demografía, economía y sociología.

Sobre todo, porque una de las características de las migraciones en la actualidad es que no se limita a un flujo de personas en movimiento, sino que implica un importante flujo de bienes materiales y simbólicos, como pueden ser capitales, información, e incluso valores culturales que generan una interconexión entre las sociedades de origen y destino, y que en la práctica funciona como un sistema integrado. Si bien, ya se ha expuesto previamente la función de las migraciones en la acumulación y reproducción del capital, esta sección del documento se centra en evidenciar las contradicciones que resultan de la complementariedad entre las tres dimensiones señaladas.

En la actualidad, el envejecimiento de la población junto al descenso de la fecundidad en el marco de la Segunda Transición Demográfica, ${ }^{14}$

14 Se refiere a la dinámica demográfica en las sociedades europeas de fines del siglo XX, que experimentaron un continuo descenso en los niveles de fecundidad, llegando en algunos casos a estar incluso por debajo de los niveles que asegurarían la reproducción demográ- 
hacen que tanto la dinámica económica de los países desarrollados como la reproducción social de su población dependan de los aportes demográficos que genera la inmigración. Sin embargo, los beneficios de este régimen tienen su propia contradicción, la cual se manifiesta en la transformación étnica y demográfica de las poblaciones de primer mundo; ya que los inmigrantes lejos de asimilarse plenamente a las sociedades de destino y ser vistos como una minoría demográfica, contribuyen al surgimiento de formas culturales, sociales y simbólicas alternativas, reforzando su condición de sociedades multiculturales, pero también de diferenciación étnica y desigualdad social (Canales, 2015: 113). Esta situación plantea un importante dilema: asegurar el proceso de reproducción demográfica, a través de una política migratoria de apertura y tolerancia a la inmigración, o bien la adopción de una política radical de control y freno, a riesgo de entrar en un proceso de inestabilidad demográfica con importantes implicaciones económicas, políticas y sociales.

Aunado a estos cambios demográficos, las economías desarrolladas están experimentando una serie de transformaciones económicas y productivas. Sin embargo, lo más relevante de este proceso es la creciente polarización de la estructura de las ocupaciones, ya que junto al auge de empleos y puestos de trabajo altamente calificados derivados de la economía del conocimiento, surgen y se expanden actividades y ocupaciones de bajo nivel productivo, altamente precarias y vulnerables, pero que crecen a la par de las primeras (Sassen, 2007; Aragonés \& Salgado, 2011). En este contexto de polarización, se van configurando nichos laborales —en los cuales suelen insertarse preferentemente los trabajadores inmigrantes-, como el servicio doméstico, la industria del cuidado, mantenimiento, entre otros, que contribuyen a la configuración de un sistema global de reproducción social de las clases medias y altas de las sociedades avanzadas y en particular de sus patrones de comportamiento y estilos de vida.

En este sentido, se trata de un proceso contradictorio. Es decir, la pobreza y precariedad de los trabajadores migrantes, no es resultado de su exclusión del mercado de trabajo, sino que es consecuencia de la forma en la que son integrados en el mundo laboral. Por lo tanto, en el actual marco de desregulación económica y flexibilidad laboral, es la modernización y globalización la que genera y reproduce sus pro-

fica. Las causas de estos cambios se sustentan en una tendencia a la individuación de los procesos sociales y familiares característicos de las sociedades contemporáneas. 
pias formas de pobreza, marginación y exclusión (Canales, 2013: 24). Así, el dilema actual de los países centrales es que para reproducirse económica y socialmente necesitan transformarse demográficamente y una de las principales alternativas es la inmigración.

Derivado del análisis anterior, es factible sostener que el origen estructural de la migración contemporánea no radica fundamentalmente en las condiciones de pobreza y marginación de los países de origen, sino en la prevalencia de un estilo de desarrollo y de integración al patrón de acumulación global que acentúa las desigualdades sociales y asimetrías económicas entre los países. Por consiguiente, la migración cambia sus características de acuerdo con los requerimientos de los patrones de acumulación, adquiriendo funciones determinadas y características enmarcadas en la permanencia y el funcionamiento del sistema (Aragonés \& Dunn, 2005: 46). Aspecto que se analiza a continuación.

\section{EL PATRón DE ACUMULACIÓN COMO CATEGORÍA DE ANÁLISIS}

Los desplazamientos humanos han existido a la par del surgimiento del hombre sobre la Tierra modificando la geopolítica del mundo. Sin embargo, aquellos que se producen a partir del desarrollo del sistema capitalista, tienen un comportamiento específico asociado a las transformaciones de las distintas fases de acumulación. Con las primeras manifestaciones del capitalismo, se desarrolla un proceso desigual en el que la expansión económica a nivel internacional se instala en espacios heterogéneos a nivel social y demográfico (Aragonés, 2000: 15, 22). Este fenómeno histórico se repetirá, con diversos matices y temporalidades en todos aquellos países que avanzan hacia el capitalismo, dando paso a lo que José Valenzuela (1990: 65) denomina patrón de acumulación y define como:

Una forma históricamente delimitada de la reproducción capitalista, lo que supone una unidad específica entre formas específicas de acumulación, producción y realización de la plusvalía y (en América Latina) una articulación específica del polo dominante interno con las formas precapitalista (y capitalistas) subordinadas, y también una articulación determinada con los centros capitalistas dominantes.

Cabe señalar que, dicho fenómeno implica un conjunto de aspectos básicos que se vinculan entre sí de un modo específico, entre los que destacan: 
a) La forma que asume el sistema de fuerzas productivas en el periodo correspondiente.

b) La articulación de los procesos de producción, distribución, utilización y realización de la plusvalía.

c) Las formas que asume la heterogeneidad estructural.

d) Las formas de la dependencia estructural.

e) La forma en la que opera la variable política, en función de la fracción clasista hegemónica y cuáles son los mecanismos de dominación primordiales (Valenzuela, 1997: 27).

Como se puede observar, analizar el proceso de acumulación en un espacio y tiempo determinado no es una cuestión precisamente económica, sino también un problema político. Por consiguiente, la utilización del concepto patrón de acumulación permite articular el papel de las crisis cíclicas y estructurales del capitalismo con el proceso por el cual el capital pasa de una fase a otra en el proceso de valorización. De acuerdo con Valenzuela $(1990 ; 1996 ; 1997)$ es posible identificar los siguientes patrones a lo largo de la historia:

a) El modelo primario exportador que se produjo desde mediados del siglo XIX hasta la gran crisis de los años treinta y se caracterizó porque la fuente principal del dinamismo económico se centraba en la demanda exterior de productos primarios. La Primera Guerra Mundial, la gran crisis y la Segunda Guerra Mundial marcarían la transición hacia un nuevo periodo, conocido como industrialización sustitutiva de importaciones.

b) El patrón secundario exportador se caracterizó por un avance del proceso de industrialización hacia una fase más compleja, se elevaron los niveles y ritmos de crecimiento de la productividad del trabajo, aumentó el grado de monopolio y la distribución del ingreso regresiva, todo esto con un alto grado de heterogeneidad y desestructuración clasista.

c) El patrón neoliberal surge como consecuencia de la crisis de la deuda de 1982 y la adopción de programas de ajuste estructural. Se caracteriza por la desregulación y relativa pasividad estatal, la apertura extrema, explotación y despilfarro, cuasi-estancamiento e inestabilidad y el capital financiero como la fracción hegemónica. 
Bajo esta perspectiva Aragonés y Salgado (2014: 234) señalan que "a diversos patrones de acumulación correspondieron diversos patrones migratorios que responden a los requerimientos de la acumulación capitalista y las exigencias de los mercados laborales". Es decir, la migración de trabajadores es una de las tantas formas de reasignación de excedentes a favor del centro si se considera que en el sistema capitalista existe una relación contradictoria entre centro-periferia, de tal suerte que a la periferia se le asignan formas de extracción y traslados de excedentes a favor del centro. Como resultado, los patrones migratorios ${ }^{15}$ modifican sus características en respuesta a las necesidades de reproducción del capital.

Incorporar la dimensión histórica al análisis, permite explicar porque los flujos de personas se contraen o expanden en períodos determinados como resultado de las contradicciones que genera el sistema capitalista, en su afán de obtener la mayor tasa de ganancia posible. Aragonés (1999; 2000) (Argonés y Salgado, 2014) siguiendo a Marx indica que, es posible identificar tres patrones migratorios, los cuales se han transformado en función de las necesidades de acumulación capitalista: las migraciones clásicas comprendidas entre los siglos XIXXX; aquellas surgidas en el fordismo y la sustitución de importaciones (1945-1970) y la globalización; y finalmente, las producidas por el neoliberalismo (1980-2009).

\section{MIGRACIÓN Y ETAPAS HISTÓRICAS DE ACUMULACIÓN CAPITALISTA}

El patrón migratorio asociado a la Revolución Industrial tuvo lugar a finales del siglo XIX con las migraciones transoceánicas, cuyas consecuencias fueron decisivas para el desarrollo del capitalismo. A partir de este momento, se inician los procesos de internacionalización del capital con la ampliación de la frontera agrícola, al instalarse en regiones prácticamente deshabitadas, pero con una gran riqueza mineral y agrícola. Una cantidad considerable de regiones se incorporaban al mercado mundial, produciéndose una nueva distribución geográfica de la población, conforme a las reglas del juego de Europa (potencia hegemónica de la época) al establecerse la primera gran división internacional de trabajo (Aragonés, 2000: 23, 44).

15 Para una revisión teórica sobre la conceptualización del patrón migratorio consultar el trabajo "Nuevo patrón migratorio bajo el contexto de la crisis" de Aragonés y Salgado (2014). 
En consecuencia, "las migraciones desde la Europa que se industrializa continúan hasta que el crecimiento vegetativo se atenúa y el proceso de industrialización se consolida" (Rodríguez, 1985: 31). Estos desplazamientos fueron útiles para disminuir la presión demográfica y facilitar el desarrollo de la industria. Posteriormente, Europa recobra nuevamente el equilibrio entre población y recursos, mientras que otros países europeos de emigración no alcanzarán dicho equilibrio; de aquí que las migraciones continúen años más tarde, pero en otras direcciones. En los países de destino el panorama también será distinto, algunos se desarrollarán y seguirán siendo destino de migraciones, otros escasamente mejorarán, y con la llegada de la explosión demográfica se convertirán en países de emigración (Rodríguez, 1985: 33).

Años más tarde, con el surgimiento del fenómeno del subdesarrollo y la consolidación del imperialismo, se configura un nuevo patrón de acumulación: el capitalismo monopolista, caracterizado por un comportamiento migratorio con tendencia sur-norte. A diferencia de los desplazamientos Este/Oeste del periodo anterior, los flujos migratorios se originan en las regiones menos desarrolladas, atraídos por el capital que circula entre las naciones desarrolladas. En esta época, la incorporación de mano de obra extranjera fue el principal instrumento que les permitió a los polos desarrollados superar las dificultades de sus mercados laborales. La mano de obra migrante se insertó en las ramas de producción de escasa o nula calificación, aspecto que fue clave para el incremento de los niveles de productividad (Aragonés, 1999; Aragonés, 2000).

En el periodo de la posguerra (1945-1970) conocido como la "era de oro del fordismo" se produjo un movimiento migratorio con una fuerte presencia intracontinental europea y otro contingente importante, denominado "sustrato colonial" hacia los antiguos imperios (el Reino Unido y Francia), con desplazamientos cortos entre países fronterizos compuestos básicamente por trabajadores no calificados, varones jóvenes y campesinos. Un hecho bastante peculiar, es que se produjo una competencia entre países, fundamentalmente europeos, que orilló a los gobiernos a pactar con las economías en desarrollo el reclutamiento de sus trabajadores en diferentes formas contractuales:

Trabajador Visitante, Trabajadores Huésped, Programa Bracero, Gastarbeiter en Alemania, etcétera. Durante 25 años se mantuvo un flujo 
prácticamente continuo de fuerza de trabajo de los polos de menor desarrollo a los industrializados (Aragonés, 1999: 730).

A partir de la recesión mundial de los años setenta, la conflictividad de los desplazamientos migratorios se comienza a manifestar en las regiones receptoras. Las acciones recesivas se vuelcan contra la fuerza de trabajo extranjera, el eslabón más débil de la cadena laboral (Aragonés, 1999: 732). El derrumbe del modelo fordista derivó en nuevas condiciones para la mano de obra migrante, ahora enmarcada en las necesidades de la reestructuración productiva. De acuerdo con Alejandro Dabat (2002: 53) esta nueva fase de acumulación asoció la internacionalización con depresión como un nuevo fenómeno estructural. En este escenario contradictorio se manifiesta el racismo y la xenofobia, los migrantes son considerados los grandes culpables de los problemas de desempleo y el descenso en los niveles de vida de los trabajadores. A partir de ese momento se comienza a especular sobre un posible caos migratorio: nacionalismo, proteccionismo y freno a la internacionalización, fueron las primeras medidas adoptadas en respuesta a la crisis. Posteriormente, algunas de las estrategias que se promovieron, fue la cancelación de los acuerdos firmados entre algunos países, los programas de repatriaciones o en su caso exportaciones, y la promulgación de leyes antiinmigrantes. Aspectos que orillarían a los países tradicionalmente receptores de migrantes a intentar un cierre de sus fronteras (Aragonés, 1999: 732).

La reestructuración productiva de los años ochenta supuso un cambio de los procesos industriales, la fuerza de trabajo se enfrentó a nuevos requerimientos de calificación y a la eliminación de ciertos puestos de trabajo ocupados por inmigrantes. Aspecto que derivó en el establecimiento de un mercado de trabajo dual (Micolta, 2005; Gómez, 2010): un primer nivel ocupado por trabajadores calificados y de tiempo completo, y el otro, conformado por jóvenes, mujeres e inmigrantes en condiciones laborales y salarios inferiores. Así, los países en desarrollo pasaron a ser la sede de las llamadas fábricas para el mercado mundial, lo que condujo a la consolidación de un mercado mundial de fuerza de trabajo y un mercado mundial de centro de producción.

Tal es el caso de las ciudades globales, en las cuales se ha registrado un aumento considerable en la intensidad y la magnitud de las transacciones, a través de los mercados financieros, el comercio de servicios y las inversiones (Sassen, 2008). A partir de los años noventa, graves crisis golpearon a determinados países y las enormes dificultades 
para superarlas pusieron en jaque al sistema. La caída de las bolsas del mundo entero, el fracaso de Rusia para transitar hacia el capitalismo o la recesión de Japón supuso para algunos autores el fin del capitalismo global (Dietrich, 1998, citado por Aragonés, 2000: 28).

Dos décadas más tarde, una nueva crisis económica de características globales se produjo entre 2007 y 2008 . En respuesta al incremento del desempleo sin precedentes que se produjo en las principales potencias económicas, los flujos migratorios de trabajadores de baja calificación disminuyeron considerablemente. Esta situación, sugiere la conformación de un nuevo patrón migratorio que, si bien por su actualidad es difícil de caracterizar en toda su complejidad, comienza a mostrar claros indicios del enorme peso que se les otorga a los trabajadores migrantes altamente calificados, al ser considerados un elemento importante para favorecer la superación de la crisis en los países receptores, particularmente en Estados Unidos (Aragonés y Salgado, 2014: 233).

Probablemente el capitalismo se encuentra atravesando por un momento de turbulencias estructurales, ya sea de carácter económico, financiero o civilizatorio. Sin embargo, no hay elementos que sugieran una transición hacia nuevos modos de producción y mientras se siga desarrollando bajo las mismas condiciones, la migración seguirá siendo un factor productivo importante que le permita sortear las dificultades a las que se enfrenta en la actualidad. Por lo tanto, un elemento adicional a incorporar en el análisis del fenómeno migratorio, son las crisis, ya que son el punto de inflexión que marcan el surgimiento de nuevas etapas de acumulación y, por consiguiente, la migración de trabajadores se expande o contrae en función de los requerimientos del capital. Para dar paso a la siguiente fase del análisis es preciso señalar que, factores de corto plazo como pueden ser los auges o las crisis económicas, tanto en los países de origen como en los de destino influyen en la magnitud y dirección de los flujos migratorios. En el último cuarto de siglo la globalización ha aumentado la frecuencia de las crisis económicas. Sin embargo, el impacto sobre los flujos dependerá de diversos factores, como se señala a continuación.

\section{LOS EFECTOS DE LA CRISIS ECONÓMICA EN LAS MIGRACIONES}

La configuración de países de origen y de destino para los migrantes internacionales ha cambiado considerablemente respecto a sus patrones históricos anteriores. En las últimas tres décadas, algunos países 
pasaron de ser naciones de emigración neta a ser países de destino para personas provenientes principalmente de América Latina, Asia, África, Europa del Este y Rusia; generalmente regiones con lento crecimiento económico, altos niveles de desempleo e inestabilidad política. Sin embargo, las crisis económicas de fines de la primera y segunda década del siglo XXI indican que los ciclos económicos que dan origen a los flujos migratorios también pueden afectar a economías desarrolladas (Solimano, 2013: 22). Tal es el caso de la disminución de los flujos migratorios hacia Estados Unidos, Europa y otras economías al ser gravemente afectadas por la crisis estructural de 2007-2008. Situaciones similares se observaron con anterioridad en diversos periodos de la historia, particularmente en años de guerra, inestabilidad y crisis.

En la actualidad, existen variadas actitudes hacia el fenómeno migratorio por parte de los gobiernos y de la población: desde una relativa apertura y tolerancia hacia los inmigrantes, generalmente en tiempos de bonanza económica, buenas oportunidades de empleo y de internacionalismo en las ideas dominantes, hasta la renuencia, hostilidad y xenofobia durante períodos de recesión, desempleo e inseguridad financiera, donde incluso se les alienta a los migrantes foráneos a regresar a sus países de origen (Solimano, 2013: 21). Por lo tanto, hay una gran contradicción entre las políticas migratorias y el efecto de la migración en el mercado de trabajo, donde la actual globalización ha aumentado la movilidad internacional de los bienes y el capital, pero no en forma articulada las migraciones internacionales.

Para Jorge Durand (2017: 6) "las políticas migratorias suelen ser pendulares, por no decir bipolares”. Cuando la economía se expande, los inmigrantes son considerados la solución perfecta por ser mano de obra barata, laboriosa y eficiente. En cambio, cuando los grandes proyectos de nación fracasan, los inmigrantes se convierten en una carga, son percibidos como innecesarios, tratados como desechables y conminados a que regresen a su país de origen. En este sentido, es preciso evidenciar la relación que existe entre los ciclos económicos y los ciclos migratorios, tanto en los periodos de crecimiento como en los de recesión y estos a su vez con los contextos económicos y las políticas migratorias (D’Anglejan, 2009: 14).

Generalmente, en los periodos de crecimiento económico los migrantes salen de sus países de origen en búsqueda de oportunidades laborales y económicas en las regiones económicamente dinámicas del mundo, y ante condiciones macroeconómicas adversas como las crisis 
económicas, los incentivos a la emigración disminuyen y los flujos se reducen. La historia del siglo XX permite advertir con claridad que las políticas nacionalistas y las medidas restrictivas de los movimientos migratorios por parte de los Estados son características de los tiempos de crisis. Al respecto, James Gould (1979) señala que:

La Primera Guerra Mundial supuso el primer retraimiento importante de los desplazamientos internacionales de población que caracterizaron la segunda mitad del siglo XIX y las primeras décadas del XX pues, no sólo los países beligerantes por razones militares y de integridad territorial, sino también los neutrales por motivaciones económicas o de falta de seguridad en los transportes, levantaron en estos años de conflicto algún tipo de barrera para cerrar o frenar la salida y entrada de población civil.

Finalizada la guerra, los países tendieron a evitar el ingreso de trabajadores foráneos y a emplear criterios cada vez más excluyentes para seleccionar a los inmigrantes que habrían de traspasar sus fronteras; los gobiernos hicieron lo posible para reservar a sus connacionales los puestos de trabajo disponibles. De acuerdo con Solimano (2013: 116) "los años de entreguerras fueron aislacionistas, anti-comercio y anti-migración, a causa de un sentimiento de rechazo a la globalización", prácticamente estos años fueron poco amistosos con la movilidad del trabajo y el capital.

Sin embargo, la Gran Depresión de los años treinta supuso una verdadera ruptura en la tendencia general de largo plazo del fenómeno migratorio internacional. A pesar de que el cierre definitivo de las fronteras era impracticable, se impusieron requisitos hasta entonces no contemplados en las políticas públicas como la obligatoriedad del uso de pasaportes, los visados de entrada, certificados de salud y de buena conducta, posesión de recursos económicos, garantía de trabajo asegurado, entre otros aspectos (Gil, 2010: 244).

El caso más emblemático fue el de Estados Unidos, las grandes leyes que establecieron limitaciones drásticas a los ingresos de personas se ratificaron durante la recesión económica, poniendo fin a una época que ciertos académicos identifican como la era de las migraciones libres. Las autoridades estadounidenses cerraron las fronteras y redujeron las entradas de inmigrantes a un nivel casi nulo, paralelamente expulsaron a casi dos millones de trabajadores entre residentes legales e inmigrantes irregulares. Asimismo, muchos decidieron retornar por cuenta propia huyendo de las presiones y las amenazas xenófobas, 
dando lugar a un episodio de la historia estadounidense denominado como éxodo (D’Anglejan, 2009; Gil, 2010).

Posteriormente, la gran recesión de los años 70's provocó que los países industrializados, que en años anteriores habían admitido a inmigrantes, hicieran esfuerzos por frenar los flujos migratorios de llegada y, además, promovieran el retorno de los inmigrantes que se encontraban en sus territorios (Herrera y Montoya, 2015: 76). A partir de 1973, la migración indocumentada hacia los países industrializados se incrementó junto con el aumento de los ingresos de refugiados y solicitantes de asilo hasta mediados de los ochenta, situación que contribuyó a incrementar el control sobre las fronteras. Es en esta coyuntura que algunos países como Estados Unidos, Francia y Alemania aplicaron estrategias para regular la migración que incluyeron:

La puesta en práctica de sanciones contra los patrones, los programas de legalización o amnistía, los programas de admisión de trabajadores extranjeros temporales, las políticas de asilo, las medidas en contra del contrabando y el tráfico de personas, las medidas de integración regionales y las estrategias para evitar la migración ilegal al impulsar un desarrollo sustentable a través de inversión o ayuda en el extranjero (Castles y Miller, 2004: 121).

Desde principios de los años 90's, la mayoría de los países de la Organización para la Cooperación y el Desarrollo Económicos (OCDE) han modificado sus leyes y procedimientos de entrada, aprobando medidas como:

Controles fronterizos más estrictos, requerimientos para las visas, revisiones de los documentos de identidad, inspecciones en los lugares de trabajo, técnicas para la detección de documentos falsos y castigos más severos para aquellos que sean sorprendidos infringiendo las reglas (Castles y Miller, 2004: 148).

Finalmente, la crisis estructural que surgió en Estados Unidos y se extendió a la mayoría de los países capitalistas del planeta, planteó nuevas condiciones para los flujos migratorios. Los sectores laborales con alta concentración de migrantes, como la construcción, el comercio y las manufacturas fueron de los más afectados, la desaceleración de estos sectores económicos se tradujo en una elevación de la tasa de desempleo a nivel nacional, que para el año 2008 alcanzó la cifra 
de 10.6 por ciento, nivel no registrado desde 1983 (BBVA Research, 2010: 5).

Los costos de la migración se incrementaron y el ambiente de crisis trajo de vuelta el aumento de las deportaciones y acciones anti-inmigrantes; durante la administración del presidente Barak Obama se llevó a cabo un programa de deportaciones, estrategia similar a la de otros momentos históricos, la cual alcanzó números alarmantes (cerca de dos millones de personas, la mayoría mexicanos) y trajo consigo consecuencias lesivas para las familias que tuvieron que enfrentar esas separaciones. Asimismo, vale la pena destacar el retorno de grandes contingentes de trabajadores que ante la crisis optaron por volver a sus países de origen (Aragonés, 2016: 10).

Es preciso señalar que la debacle económico-financiera de 2008 solamente fue el síntoma más visible de una recomposición de los patrones tradicionales de la migración, que ya se venía gestando en años anteriores. Esta coyuntura económica simplemente se sumó al incremento de políticas restrictivas que se implementaron en diversos países europeos y particularmente en Estados Unidos a partir de 2001, y que en la actualidad se expresa a través del endurecimiento de la seguridad fronteriza, la restricción de la naturalización y la residencia de largo plazo, la ampliación del marco legal para las deportaciones y la promoción del retorno.

Como ya se ha señalado, esta crisis global establece el inicio de un nuevo patrón de acumulación y un nuevo patrón migratorio que responde a las nuevas exigencias de los mercados laborales, pues las grandes crisis son el punto de inflexión hacia nuevas etapas productivas. En otros momentos históricos, las deportaciones, los cierres fronterizos, las reducciones drásticas de programas temporales de empleo y de reunificación familiar, eran estrategias empleadas para afrontar las crisis; en la actualidad se observa una estrategia diferente, sobre todo con los migrantes calificados, que lograron mantener una tendencia al alza en varios países, incluso durante este período. Por el contrario, esta medida afectó a los flujos migratorios con menores grados de cualificación.

\section{CONCLUSIONES}

La migración es un fenómeno dinámico y complejo que se adscribe a la globalización como un proceso que abarca un sinnúmero de contextos, situaciones y experiencias. Por ello, se plantea la necesidad imperiosa 
de comprender el fenómeno migratorio como una totalidad y no sólo como una sucesión de hechos aislados, puesto que está presente a nivel global en distintas dimensiones, y como tal, el análisis de los procesos globales debe incluir contextos históricos, estructuras, prácticas e instituciones. Desde esta perspectiva, la migración deja de ser un fenómeno externo para constituir un componente clave en la reproducción y transformación de la sociedad global, hecho que permite replantear su construcción como objeto de estudio y problematización; ya que la mayoría de los enfoques se centran en las causas, funcionalidad y consecuencias de la migración, y la reflexión sobre los aspectos estructurales queda relegada a un segundo plano.

En este orden de ideas, lo que se plantea es que el origen estructural de la migración contemporánea no radica tanto en las condiciones de subdesarrollo y pobreza en los países de origen, sino en la prevalencia de un estilo de desarrollo y de integración al patrón de acumulación global que acentúa las desigualdades sociales y asimetrías económicas entre los países; y que particularmente, se desencadena por las transformaciones en la estructura económica, laboral y demográfica de las sociedades de destino. Por lo tanto, analizar la migración desde la perspectiva de reproducción de la sociedad permite por un lado, evidenciar las contradicciones y tensiones que surgen de los procesos de acumulación, tanto en la forma social como productiva (reproducción demográfica, reproducción de la economía y el capital, reproducción de la estratificación y desigualdad sociales ); y por el otro, resaltar un componente central del enfoque estructural y del marxismo en particular: la contradicción como motor de acumulación.

Adicionalmente, es preciso señalar que el desarrollo del capitalismo no ha sido un proceso lineal, ya que siempre ha estado sometido a fluctuaciones cuya principal expresión son las crisis. Sin embargo, se considera que las crisis estructurales (la Gran Depresión, la recesión de los años 70's del siglo XX y la crisis estructural de 2007-2008) son el punto de inflexión que marca el surgimiento de nuevas etapas de acumulación y de patrones migratorios, que responden a los requerimientos del capital y a las exigencias de los mercados laborales. Por consiguiente, la movilidad internacional del trabajo y del capital no ocurre en un vacío institucional e histórico, son fenómenos vinculados entre sí, como muestra la historia económica mundial del último siglo y medio. Ambos fenómenos deben ser analizados en forma conjunta, ya que la movilidad está estrechamente ligada a los regímenes de polí- 
tica económica de los países, el grado de apertura, los ciclos económicos y la prevalencia de sentimientos internacionalistas versus instintos nacionalistas en la población, entre otros factores.

En el último cuarto de siglo la globalización ha aumentado la frecuencia de las crisis económicas y una pregunta clásica consiste en saber si las crisis son cíclicas; el sentido común sugiere que ninguna crisis es idéntica a otra. No obstante, existen elementos comunes y mecanismos de propagación similares que vale la pena señalar: I) Generalmente las crisis suelen estar precedidas de una fase de rápida acumulación de deuda por parte de las personas, las empresas o el gobierno. II) Las crisis se propagan más fácil entre países cuando los mercados financieros están integrados internacionalmente. III) Las crisis tienden a ocurrir cuando existen relaciones cercanas entre políticos, legisladores, banqueros y grandes intermediarios financieros, lo que dificulta aprobar una legislación financiera adecuada y/o aplicar la legislación existente. IV) El cese de pagos de la deuda y las reprogramaciones forzosas han sido características comunes de muchas crisis financieras a través de la historia (Solimano, 2013: 112,114).

La crisis global de 2008 marcó el inicio de un nuevo patrón de acumulación y un nuevo patrón migratorio que se caracteriza por las transformaciones de las tecnologías de la información y la economía del conocimiento que afecta a los sistemas productivos, y por ende, a los requerimientos de mano de obra, la cual es cada vez más polarizada. Esta situación ha provocado una reconfiguración de los flujos migratorios a nivel mundial y una competencia global por los talentos, donde se prefiere la inmigración altamente calificada (ya que los contingentes internos son insuficientes) y de carácter temporal, mientras que los flujos migratorios con menores grados de calificación van a la baja. Sin embargo, ambos flujos responden a la lógica del capital que requiere aplicar flexibilidad y desregulación laboral, debido al entorno de enorme competitividad que se vive. 


\section{REFERENCIAS BIBLIOGRÁFICAS}

Aragonés, A. (1999). El fenómeno migratorio en el marco de la globalización. Comercio Exterior, 49(8), pp. 727-738.

Aragonés, A. (2000). Migración internacional de trabajadores: una perspectiva histórica. México: Plaza y Valdés.

Aragonés, A. (2016). Introducción. En: A. Aragonés, ed. La reciente crisis financiera y el debate sobre migración y desarrollo. Propuestas para América Latina y México.. México: UNAM - Instituto de Investigaciones Económicas, pp. 9-22.

Aragonés, A. y Dunn, T. (2005). Trabajadores indocumentados y nuevos destinos migratorios. Política y Cultura, Issue 23, pp. 43-65.

Aragonés, A. y Salgado, U. (2011). Mercados de trabajo en la economía del conocimiento y el fenómenos migratorio. El caso de Estados Unidos (1990-2006). En: A. Aragonés, ed. MERCADOS DE TRABAJO Y MIGRACIÓN INTERNACIONAL. México: UNAM, Instituto de Investigaciones EconómiCAS, pp. 79-118.

Aragonés, A. y Salgado, U. (2014). Nuevo patrón migratorio bajo el contexto de la crisis . En: Crisis económica y migración. ¿Impactos temporales o estructurales? México: UNAM, Instituto de Investigaciones Económicas, pp. 231-264.

Artola, J. (2016). Nuevas tendencias migratorias en los albores del siglo XXI. En A. Aragonés, ed. La reciente crisis financiera y el debate sobre migración y desarrollo. Propuestas para América Latina y México. Primera ed. México: Universidad Nacional Autónoma de México, Instituto de Investigaciones Económicas, pp. 23-40.

BBVA Research. (2010). Situación Migración México. Mayo.

Canales, A. (2013). La migración en la reproducción de la sociedad global. Migración y Desarrollo.

Canales, A. (2015). E PUR SI MUOVE: Elementos para una teoría de las migraciones en el capitalismo global. Primera ed. México: Miguel Ángel Porrúa.

Castles, S. y Miller, M. (2004). La era de la migración Movimientos internacionales de población en el mundo moderno. México: Miguel Ángel Porrua, UAZ, Cámara de Diputados LIX Legislatura, Fundación Colosio, Secretaría de Gobernación, Instituto Nacional de Migración.

D’Anglejan, S. (2009). Migraciones internacionales, crisis económica mundial y políticas migratorias. ¿Llegó la hora de retornar? Oasis, Issue 14.

Dabat, A. (2002). Globalización, capitalismo actual y nueva configuración espacial del mundo. En: Basave, ed. Globalización y alternativas incluyentes para el siglo XXI. México. Miguel Ángel Porrua / UNAM / UAM, pp. 4-88. 
Delgado, R. y Márquez, H. (2012). Desarrollo Desigual y Migración Forzada. Una Mirada desde el Sur Global. Primera ed. México: Universidad Autónoma de Zacatecas, UNESCO, RIMD, Miguel Ángel Porrúa.

Delgado, R., Márquez, H. \& Puentes, R. (2013). Migración, desarrollo y derechos humanos. Elementos para replantear el debate. En A. Aragonés, ed. Migración y desarrollo. Debates y propuestas. México: Universidad Nacional Autonoma de México, IIEc, pp. 109-146.

Durand, J. (2017). La inmigración como amenaza en Estados Unidos. En: Anuario CIDOB de la Inmigración 2017. s.l.:CIDOB, pp. 33-49.

Gil, A. (2010). Crisis, xenofobia y repatriación. Los inmigrantes españoles en la ciudad de México, 1910-1936. Stud. hist., H. ${ }^{a}$ cont., Issue 28, pp. 239273.

Gómez, J. (2010). La migración internacional: teorías y enfoques una mirada actual. Semestre económico, enero-junio, 13(26), pp. 81-100.

Gould, J. (1979). European Inter-Continental Emigration, 1815-1914: Patterns and Causes. The Journal of European Economic History, 8(3), pp. 593679.

Harvey, D. (2003). Espacios de Esperanza. Madrid: Ediciones Akal S.A..

Harvey, D. (2006). Spaces of global capitalism. UK/USA: Verso.

Herrera, M. y Montoya, E. (2015). Migración de retorno: una interpretación teórica y conceptual frente a las cifras de migrantes retornados de Estados Unidos a México. En: E. Montoya \& M. Nava, edits. Migración de retorno en América Latina. Una visión multidisciplinaria. México: Universidad Autónoma de Sinaloa: Juan Pablos Editor, pp. 75-108.

Hjorth, S. (2011). Espacio y flujos migratorios trasregionales en el marco de la globalización. En A. Aragonés, ed. Mercados de trabajo y migración internacional. México: UNAM, Instituto de Investigaciones Económicas, pp. 311-340.

Lacy, W. (2018). Las Tres Mega Tendencias del Siglo XXI. Consltado el 13 de Noviembre de 2018 en https://onuhabitat.org.mx/index.php/las-tresmega-tendencias-del-siglo-xxi

Martinez, P. (2000). La migración internacional y el desarrollo en la era de la globalización e integración: temas para una agenda regional. Población y Desarrollo, Issue 10.

Marx, K. (2000). El capital. Crítica de la economía política. Madrid: Akal.

Micolta, A. (2005). Teorías y conceptos asociados al estudio de las. Trabajo Social, Issue 7, pp. 59-76.

Rodríguez, J. (1985). Migraciones exteriores, transición demográfica y proceso de desarrollo. REIS, 32(85), pp. 27-42. 
Sassen, S. (2003). Los espectros de la globalización. Primera edición en español ed. Buenos Aires: Fondo de la Cultura Económica Argentina.

Sassen, S. (2007). Una sociología de la globalización. Madrid: Katz Editores.

Sassen, S. (2008). Actores y espacios laborales de la globalización. Papeles, Issue 101, pp. 33-51.

Solimano, A. (2013). Migraciones, capital y circulación de talentos en la era global.. Chile: Fondo de la cultura económica.

Valenzuela, J. (1990). ¿Qué es un patrón de acumulación?. México: Facultad de Economía UNAM.

Valenzuela, J. (1996). Estrategias de desarrollo: vigentes y alternativas. Iztapalapa, pp. 129-156.

Valenzuela, J. (1997). Cinco dimensiones del modelo neoliberal. Política y Cultura, Issue 8, pp. 9-38.

Wallerstein, E. (2002). Un mundo incierto. Buenos Aires: Libros del Zorzal.

World Bank. (2016). Skills \& Innovation Policy. Consultado el 10 de Diciembre de 2018 en http://go.worldbank.org/AW9KZWJB10

World Bank. (2018). Groundswell : Preparing for Internal Climate Migration. Consultado el 10 de Diciembre de 2018 en https://openknowledge. worldbank.org/handle/10986/29461

\section{RESUMEN CURRICULAR DE LA AUTORA}

\section{Carmen Lilia Cervantes Bello}

Doctorante en Estudios Latinoamericanos de la Universidad Nacional Autónoma de México (UNAM), Maestra en Política Económica Internacional por la Universidad de Belgrano (Argentina). Profesora Investigadora del Departamento de Economía y Negocios de la Universidad del Caribe. Líneas de generación y aplicación del conocimiento: Migración Internacional y Globalización e Integración Regional. Integrante del cuerpo académico consolidado "Economía y Sociedad". Dirección electrónica: ccervantes@ucaribe.edu.mx 